\title{
Altered Lipid Profile is a Risk Factor for the Progression Andrecurrenceof COVID-19: From Two Retrospective Cohorts
}

Hui Jin

Wuhan Third hospital

Junji He

Public health Clinical Center of Chengdu

Chuan Dong

Sichuan Academy of Medical Sciences and Sichuan provincial People's Hospital

Luhong Cao

Sichuan Academy of Medical Sciences and Sichuan provincial People's Hospital

Xing Qi

Public Health clinical center of Chengdu

Tiande Huang

The second people hospital in Xindu district of Chengdu

\section{Zhiyue Ma}

Sichuan Academy of Medical Sciences and Sichuan provincial People's Hospital

Qingjia Gu

Sichuan Academy of Medical Sciences and Sichuan provincial People's Hospital

Gang He

Sichuan Academy of Medical Sciences and Sichuan provincial People's Hospital

Jiangang Fan

Sichuan Academy of Medical Sciences and Sichuan provincial People's Hospital

Bin Li

Sichuan Academy of Medical Sciences and Sichuan provincial People's Hospital

Xiao long Zhao ( $\nabla$ sczhaoxiaolong@163.com )

Department of Otolaryngology Head and Neck Surgery,Sichuan Provincial People's Hospital, University of Electronic Science and Technology of China, Chengdu, China https://orcid.org/0000-0003-2356-272X

\section{Research}

Keywords: COVID-19, dyslipidemia, recurrence, metabolic syndrome, prognosis

Posted Date: August 19th, 2020

DOI: https://doi.org/10.21203/rs.3.rs-60159/v1

License: @ (1) This work is licensed under a Creative Commons Attribution 4.0 International License. Read Full License 


\section{Abstract}

Background The COVID-19 pandemic has spread worldwide. However, the impact of lipid profile and lipid-lowering treatment on clinical endpoints in COVID-19 have not previously been investigated.

Methods In this retrospective, multicenter cohort study, we consecutively enrolled 430 adult COVID-19 patients from two Chinese hospitals (one each in Chengdu and Wuhan) admitted during February 2020 and followed-up until April 30. Demographic, metabolic profile, laboratory, treatment and clinical endpoint data including in-hospital death and recurrence of COVID-19, were collected.

Results In Chengdu patients, univariable and multivariable Cox regression showed that the low-density lipoprotein cholesterol (LDL-C) dyslipidemia on admission was associated with the recurrence of COVID-19 during the follow-up period. In Wuhan cohort, the patients with triglycerides hyperlipemia had an increased risk of in-hospital death. However, in both cohorts, statin therapy during COVID-19 course did not affect these clinical endpoints. Compared to the Chengdu cohort, the Wuhan patients tended to have more severe COVID-19 but, unexpectedly, had lower levels of serum lipid. It is of interesting to notice that the relationship between the observed biomarkers of inflammation and lipid do not match the relationship between the organ function measures and this lipid.

Conclusions The baseline dyslipidemia should be considered as a risk factor for poor prognosis and recurrence of COVID-19. The lipid level may be altered during COVID-19 course, since lipidology may be distinctly affected by both inflammation and organic damage for SARSCoV-2. Further investigation is needed on the role of use of lipid-lowering therapy among patients with COVID-19 infections.

\section{Introduction}

In December 2019, a new viral disease was identified in China, termed coronavirus disease 2019 (COVID-19), which has since spread rapidly throughout the world[1]. A novel beta-coronavirus, known as severe acute respiratory syndrome corona virus 2 (SARS-CoV-2), was identified as the COVID-19 pathogen and is known to trigger severe pneumonia and acute, often lethal, lung failure[1]. By April 14, 2020, 1.92 million confirmed cases of COVID-19 and 21,000 deaths had been reported across 195 countries[2]. In addition, many discharged COVID-19 patients had positive oropharyngeal swab tests for SARS-CoV-2 RNA during recovery[3]. COVID-19 will inevitably impose a significant burden on healthcare systems due to not only treatment but the increased need for supervision after discharge [4]. Therefore, it is important to identify risk factors for disease recurrence, which can be identified by an RT-PCR test for SARS-CoV-2 performed after discharge. It is also essential that COVID-19 patients are advised regarding how to reduce the risk of recurrence such as the life style change. To date, no studies have reported on the risk factors for recurrence of COVID-19 after discharge.

Multiple cardiovascular metabolic abnormalities included diabetes, obesity, hypertension, and dyslipidemia[5]. These are all important risk factors for cardiovascular disease, which is one of the leading causes of morbidity worldwide and is anticipated to rise substantially over the next decades[6, 7]. On admission, 20-51\% of COVID-19 patients reported at least one comorbidity, with the most common being diabetes (10-20\%), hypertension (10-15\%) and other cardiovascular metabolic comorbidities(7-40\%)[8-13]. A retrospective multicenter study conducted in China revealed that patients with these comorbidities (based on self-report) on admission experienced poorer clinical outcomes than those without comorbidities[8]. Only two studies of patients in Wuhan showed that serum lipid levels were unexpectedly lower in COVID-19 patients compared to healthy subjects[14, 15].On the other hand, it appears that lipid-lowering pharmacological interventions, in particular statin, might reduce the risk of cardiovascular complications caused by COVID-19 and might potentially have an additional antiviral activity[16]. We need to describe lipid pathological alterations in COVID-19 and what about lipid-lowering therapy and their final influence on these final results.

Here, we present the details of patients admitted to two hospitals (located in west and central China) with laboratory-confirmed COVID-19. The information reported includes primary clinical endpoints, such as in-hospital death and recurrence rates after discharge, and secondary endpoints including the intensive care unit (ICU) admission and mechanical ventilation rates. We also report the lipid risk factors identified on admission and lipid-lowing treatments associated with poor clinical endpoints.

\section{Methods}

\section{Study design and subjects}

This retrospective cohort study included two cohorts of adult subjects (aged $\geq 18$ years) recruited from the Public Health Clinical Center of Chengdu (Chengdu cohort) and the Wuhan Third Hospital (Wuhan cohort). All adult patients diagnosed with COVID-19 according to the World Health Organization (WHO) interim guidelines were screened during February and then monitored until April 30, 2020. A total of 430 adult patients were hospitalized from the two hospitals during the February. The exclusion criteria were 1) due to missing data in their 
medical records ( $n=63)$; 2 ) because the sample size was too small for statistical analysis, the in-hospital death patients from the Chengdu cohort were excluded $(n=6) ; 3$ ) patients who received treatment with lipid-lowering medications before the study in both cohorts ( $n=22)$; 4 ) patients in the study who received other lipid-lowering therapy rather than a single agent treatment of statin wereexcluded $(n=13)$. The final analysis included 326 patients with complete data for all clinical variables.

\section{Data collection and definitions}

Epidemiological, demographic, clinical, laboratory, treatment and endpoint data were collected from electronic medical records. All the data collected were reviewed by two physicians ( $\mathrm{JH}$ and $\mathrm{HJJ})$. A researcher $(\mathrm{ZXL})$ was consulted to resolve differences in interpretation between the two primary reviewers. Routine blood tests were performed to assess the blood count profile, and biochemical analysis of blood serum was conducted to determine the levels of aspartate aminotransferase (AST), alanine aminotransferase (ALT) and creatinine, as an indicator of renal and liver function. The other serum markers measured included creatine kinase, C-reactive protein (CRP), lactate dehydrogenase, myocardial enzymes and procalcitonin. All patients were assessed by chest computed tomography (CT).

Fever was defined as an axillary temperature of at least $37.3^{\circ} \mathrm{C}[17]$. Septic shock was defined according to the 2016 Third International Consensus Definition for Sepsis and Septic Shock[18]. The severity of COVID-19 was defined according to the Chinese management guideline for COVID-19 (version 7.0)[17]. The primary endpoint was the presence of SARS-CoV-2 RNA after discharge or in-hospital death. The secondary endpoint was admission to the ICU or the need for invasive ventilation. These endpoints were used as they represent the most serious outcomes of COVID-19 infection, and have been employed in previous H7N9 report[8, 19]. Comorbidities were categorized according to the organ system affected. For example, chronic obstructive pulmonary disease (COPD) and asthma were categorized as the pulmonary disease [8]. Self-reported questionnaires were used to obtain information on smoking and drinking status, comorbidities, and any other relevant aspects of the medical history. Regarding lipid-lowering therapy status, patients were classified as current or noncurrent lipidlowering therapy. Medications and dose on the specific class of lipid lowering agents were ascertained. Intensity of statin therapy was classified into high-intensity, moderate-intensity, and low-intensity, according to the 2013 ACC/AHA guidelines[20]. The indications for discharge included the absence of fever for at least 3 days, substantial improvement in both lungs based on chest CT, clinical remission of respiratory symptoms, and two throat swab samples negative for SARS-CoV-2 RNA (interval of at least 24 hours)[17]. Throat swab specimens were tested for SARS-CoV-2 RNA if any respiratory symptoms appeared, and routinely every 2 weeks after discharge for 1 month [17]. The endpoint of recurrence was defined as the presence of SARS-CoV-2 RNA after discharge. The time for the endpoint in Chengdu cohort was defined as the time of the recurrence until the follow-up deadline. The time for the endpoint in Wuhan cohort was defined as the time of the in-hospital or discharge.

\section{Anthropometric and biochemical measurements}

Body mass index (BMI) was calculated as weight in kilograms divided by height in meters squared (kg/m²). Daytime blood pressure (BP) was measured after at least 5 minutes of rest in a sitting position using a mercury sphygmomanometer. The mean of three measurements was recorded, as suggested in the American Society of Hypertension Guidelines [21]. For each subject, serum lipid profiles were obtained, including total cholesterol (TC), triglycerides (TG), high-density lipoprotein cholesterol (HDL-C), and low-density lipoprotein cholesterol (LDLC). Serum fasting glucose (FG) was measured using an $\mathrm{H}-7600$ autoanalyzer (Hitachi, Tokyo, Japan). The criteria for dyslipidemia or hyperlipidemia were TC $\geq 5.17 \mathrm{mmol} / \mathrm{L}$ or LDL-C $\geq 3.33 \mathrm{mmol} / \mathrm{L}, \mathrm{TG} \geq 150 \mathrm{mg} / \mathrm{dL}(1.7 \mathrm{mmol} / \mathrm{L})$ or HDL-C $<40 \mathrm{mg} / \mathrm{dL}(1.0 \mathrm{mmol} / \mathrm{L}) \mathrm{in}$ $\mathrm{men} /<50 \mathrm{mg} / \mathrm{dL}(1.3 \mathrm{mmol} / \mathrm{L})$ in women in accordance with the criteria of the United States National Cholesterol Education Program Adult Treatment Panel III (NCEPIII)[22].

\section{Statistical analysis}

Normal, skewed and categorical data are presented as means and standard deviations, medians with interquartile range, and percentages, respectively. Differences in baseline characteristics among disease severity subgroups were examined using the Kruskal-Wallis $\mathrm{H}$-test, oneway analysis of variance, Fisher's exact test, or the $\chi^{2}$ test according to the datadistribution. $P$-values for trends were calculated using the linear-by-linear association test for dichotomous variables.

Kaplan-Meier analysis was used to estimate survival outcomes. Univariable and multivariable Cox proportional hazards regression models were used to determine the potential risk factors for each clinical endpoint. Risk ratios (RRs) and 95\% confidence intervals (Cls) were also reported. Cox regression was considered more appropriate than logistic regression because the former can account for the potential impact of variation in follow-up times among patients. Multivariable logistic regression was used to explore the risk factors for in-hospital death, ICU admission and mechanical ventilation. Stepwise multivariate linear regression was used to identify factors associated with the lipid profile. The proportionality assumption was checked with plots of scaled Schoenfeld residuals against transformed time and a goodness-of- 
fit test using the sample size. Proportionality was not violated. All analyses were performed using SPSS software (version 20.0; SPSS Inc., Chicago, IL, USA). In all analyses, $P<0.05$ was considered statistically significant.

\section{Results}

\section{Baseline characteristics of COVID-19 patients}

The study included 326 adult COVID-19 patients positive for SARS-CoV-2 RNA (133 patients from the Chengdu cohort and 193 from the Wuhan cohort). All patients were admitted during February. The most common symptom was cough (77.5\%), followed by fever on admission (45\%). Nasal congestion (0.5\%) and nausea or vomiting (1.8\%) were reported less frequently. Compared to the Chengdu cohort patients, those in the Wuhan cohort were older, and more commonly presented with respiratory or systematic symptoms (e.g., fever, cough, sputum production, shortness of breath, fatigue or diarrhea). However, there was no difference in the rate of comorbidities between the two cohorts. The Wuhan cohort tended to have more severe COVID-19, and abnormal laboratory and chest CT results (Table 1). Interestingly, the Wuhan patients had lower lipidlevels (TC, TG, and HDL-C) and a higher fasting glucose level (Table 1). We also observed that dyslipidemia was not correlated with COVID-19 severity in either cohort (Table S1-S2). The laboratory test results of the different COVID-19 severity subgroups are shown. There was a linear trend between COVID-19 severity and the number of laboratory tests indicative of lymphopenia, inflammation [i.e. CRP, procalcitonin] and the organ function measures [i.e. AST, ALT or creatinine) .

\section{Treatment, complications and clinical endpoints of COVID-19 patients}

The treatments, complications and clinical endpoints of both cohorts are shown in Table 1. In the Chengdu cohort, the mean length of stay (in the first hospital) was 20.53 days. The longest recorded stay was 75 days. Oropharyngeal swab samples showed that $32.4 \%$ of patients were positive for SARS-CoV-2 RNA after initial discharge. The mean recurrence time from the first discharge in the Chengdu cohort was 18.22 days. Following readmission, the mean length of stay was 14.2 days. Three patients experienced COVID-19 recurrence after discharge. Overall, the mean time from the onset of illness (before admission) to first discharge was 37.11 days.

In the Wuhan cohort, more patients received treatment with antibiotics, corticosteroids intravenous immunoglobulin and mechanical ventilation compared to those in the Chengdu cohort ( $94.4 \%$ vs. $23.2 \%$; $65.2 \%$ vs. $8.9 \%$; $41.4 \%$ vs. $1.4 \%$; and $27.4 \%$ vs. $11.3 \%$, respectively). However, the patients in the Chengdu cohort had greater access to ICUs and oxygen therapy. During hospitalization, the most common complication in all patients was pneumonia (80.5\%), followed by acute respiratory distress syndrome (ARDS; $10.08 \%$ ) and septic shock (3.6\%). The prevalence rates of septic shock and pneumonia in the Wuhan cohort were significantly higher compared to those in the Chengdu cohort (5.6\% vs. $0.7 \%$ and $92.9 \%$ vs. $69.7 \%$, respectively).

In the Wuhan cohort, $28.9 \%$ of patients died during hospitalization and 140 patients were discharged. The longest delay to first admission was 60 days. The mean time from initial symptom onset to discharge was 24.25 days, while the mean time from initial symptom onset to death was 21.18 days. Follow-up of discharged patients was not possible as they were transferred to temporary hospitals for at least 14 days for further monitoring in accordance with the health policy. Telephone follow-up interviews showed that no discharged patients had died.

\section{Lipid-lowering therapy and prognostic factors}

During the study period, about $22.08 \%$ of in-patients with dyslipidemia in both cohorts received statin therapy, and majority of patients received moderate-intensity of statin, $20-40$ mg / d or equivalent statins. Furthermore, the percentage of patients treated with lipid-lowering therapy and the stating dose showed there was no significant difference between both cohorts (Table 1). Figure 1 shows Kaplan-Meier estimates of the risk of in-hospital death and recurrence of COVID-19 after discharge. The risks factors assessed included TC, TG, HDL, LDL$\mathrm{C}$ dyslipidemia present in the period and the use of stating therapy between initial symptom onset and assessment of the clinical endpoints.

In the Chengdu cohort, because the sample size of in-hospital death was too small for statistical analysis, these patients were excluded, we focused on the recurrence of COVID-19 after discharge. We chose the patients without any dyslipidemia as the control and found patients with LDL-C dyslipidemia with or without stating therapy had a significantly higher risk of COIVD-19 recurrence after discharge compared to the control patients, as revealed by a log-rank test $\left(X^{2}=10.77, p=0.0046\right)$ (Figure $\left.1 \mathrm{~A}\right)$. Previous studies reported that laboratory results (specifically ALT, lactate dehydrogenase, high-sensitivity cardiac troponin I, creatine kinase, and d-dimer levels) may be risk factors for COVID-19 prognosis and severity. Therefore, these variables were included in our multivariable Cox proportional regression model. Univariable and multivariable Cox proportional analyses adjusted for age, sex, comorbidities, and smoking and drinking status and the treatments in hospital identified LDL-C dyslipidemia as a risk factor for recurrence of COVID-19 after discharge in the Chengdu cohort (RR: 2.708, 95\% Cl: 1.283, 5.718; and RR: 2.51, 95\% Cl: 1.19, 5.29, respectively) $(p<0.05$, Table 2). Unfortunately, univariable Cox proportional 
analyses showed that the use of stating therapy had no significant effect on the recurrence after discharge (RR: $1.609,95 \%$ Cl: $0.771,3.355$, $p=0.205)$.

In the Wuhan cohort, the survival rate of patients with TG dyslipidemia with or without stating therapy was lower compared to that of patients without any dyslipidemia, based on a log-rank test $\left(X^{2}=6.907, p=0.0361\right)$ (Figure 1B). Univariable Cox proportional analysis showed that the risk of in-hospital death was found to be higher in patients with TG dyslipidemia. After adjusting for basicvariables and the treatments, TG dyslipidemia was risk factor for in-hospital death (RR: 1.92, 95\% Cl: 1.08,3.60, $p=0.016$ ) (Table 2).

We also investigated the associations of components of dyslipidemia with secondary clinical endpoints, including rates of ICU admission and invasive ventilation. Univariable and multivariable logistic regression models were used to determine risk factors. In the Chengdu cohort, univariable logistic regression showed that there was no any component of dyslipidemia associated with the requirement for mechanical ventilation. And TC or LDL-C dyslipidemia or use of stating therapy (odds ratio [OR]: $3.574,95 \% \mathrm{Cl}: 1.452,8.797, p=0.006 ; 9.849,95 \% \mathrm{Cl}$ : $2.086,46.503, p=0.004 ; 3.364,95 \% \mathrm{Cl}: 1.080,10.481, p=0.036$; respectively) was associated with a higher rate of admission to the ICU. After adjusting, only LDL-C dyslipidemia was significantly associated with ICU admission in multivariable logistic regression (OR: 10.073, $95 \% \mathrm{Cl}: 2.073,48.948, p=0.004$ ) (Table 3 ). In the Wuhan cohort, univariable logistic regression showed that only TC dyslipidemia was associated with risk of ICU admission (OR: $2.569,95 \% \mathrm{Cl}: 1.218,8.072, p=0.016)$.In a multivariable logistic regression model, only TC dyslipidemia was independently associated with ICU admission (OR: 8.147, 95\% Cl: 1.662, 39.927, $p=0.010$ ) (Table 3). Overall, the univariate and multivariable models showed that, in both cohorts, patients with dyslipidemia on admission were more likely to experience inhospital death or recurrence of COVID-19.

\section{Distinct factors associated with lipid profile on admission}

To address the effect of inflammatory and organ function biomarkers due to COVID-19 on the blood lipid levels, we used a multivariate linear regression model to determine factors independently associated with the baseline serum lipid profile.

Interestingly, the correlations of inflammatory biomarkers (CRP and procalcitonin) with lipid levels were different from those for biomarkers of organ function (AST, ALT, and creatinine). For example, after adjusting for age, sex, comorbidities, smoking and drinking status, the CRP level was negatively associated with the TC lipid level $(\beta[S E]=-0.646[0.219], p=0.005)$. However, an increased level of ALT, which indicates impaired hepatic function, was positively associated with TC lipid levels in the Chengdu cohort ( $\beta$ [SE] $=0.633[0.229], p=0.007)($ Table 4$)$. Similar correlations were seen for other lipids in the both cohorts, including TC, TG, HDL-C and LDL-C. In summary, it is important to note that we observed the inflammatory biomarkers were negatively associated with lipid level but the biomarkers of hepatic and kidney organ function were positively associated with lipid level (Table 4).

\section{Discussion}

This retrospective multicenter cohort study revealed significant differences in baseline characteristics, laboratory results on admission, severity of COVID-19, length of hospital stay, treatment types and clinical endpoints between cohorts from two different regions of China. After adjusting for demographics, self-reported comorbidities and laboratory results on admission, dyslipidemia on admission was a major risk factor for ICU admission, in-hospital death and recurrence of COVID-19 after discharge (as confirmed by detection of viral RNA in throat swab samples) in both cohorts. In both cohorts, the associations between lipid levels and inflammatory biomarkers were different to those that between lipid levels and biomarkers of hepatic and renal organ function.

Overall, our findings are in accordance with previous reports on the impact of cardiovascular metabolic comorbidities on COVID-19 patient outcomes[8]. Our multivariable analysis suggested that, in particular, dyslipidemia on admission predisposes COVID-19 patients to adverse clinical outcomes. The dyslipidemia has been reported might exacerbate significantly higher risk to atherothrombotic complications[23]. Thus, the patients with dyslipidemia should been more prone to COVID-19 with worse prognosis. In addition, the dyslipidemia was a significant risk factor for the recurrence of COVID-19 after discharge in our study. The role of lipid in viral entry has been studied for several coronaviruses including SARS-CoV[24]. Lipid presents in the cell membrane and viral envelope has been found to contribute to coronavirus replication by acting as a key component in viral entry[25]. The lipid peroxidation or lipid metabolic pathways might be involved in virus infections and viral shedding time [26, 27]. Although the above reported in vitro data suggest an essential role of lipid rafts and cholesterol in viral entry, specific confirmation in vivo is needed. In this context, these previous studies seemingly indicated the important role of statins therapy in preventing cellular entry of coronavirus can guide new therapies that may be directed SARS-CoV-2[28]. However, to the best of my knowledge, there has been almost no evidence on the use of statins in patients with SARS-CoV-2. We just found that patients from Chengdu cohort with the lipid-lowering pharmacological intervention was likely to the ICU admission $(p=0.036)$ (Table 2). This may be in part due to the fact that coronavirus might also use different receptors to enter the host cell and the possible effects of statins only via inhibition of 
special receptors such as LDL-receptors (LDL-R), resulting in there are conflicting results regarding the effects between statins therapy and dyslipidemia on the COVID-19[28, 29]. Furthermore, it has been suggested that statins act differently in ARDS patients at different status (hyper-inflammatory vs. hypo-inflammatory)[30]. Nevertheless, there is a paucity of data on this field, and thus further research is needed to elucidate the association between statin therapy and development and outcomes of COVID-19.

On another hand, a small, retrospective longitudinal study conducted in Wuhan reported a progressive and irreversible decrease in LDL-C levels over the course of the COVID-19 until death[15]. Another cross-sectional study from Wuhan demonstrated that hypolipidemia was correlated with the severity of COVID-19[14]. Similar to our study, lipid levels were lower in more severe cases of COVID-19, but there was no significant linear trend between presence of dyslipidemia and disease severity across subgroups of COVID-19, in either patient cohort. We attempted to address these conflicting results. And in our multivariate analysis of the Chengdu cohort, inflammatory biomarkers (e.g., CRP and procalcitonin) and biomarkers of impaired hepatic and renal function (elevated AST, ALT, and creatinine) on admission were negatively and positively associated with lipid levels, respectively (Table 4). This indicates that the lipid levels may decrease in the COVID-19, which was likely due to affection by the inflammation.

Inflammation and impaired organ function are two critical aspects of COVID-19 infection[31]. It has been reported that inflammation caused by viral infection may result in dyslipidemia in patients infected with dengue virus may show a decrease in serum LDL-C levels[32]. Hepatitis B patients in the cirrhosis phase have lower levels of HDL-C and LDL-C[33]. With greater severity of COVID-19, biomarkers of inflammation, and of impaired hepatic and renal function, were alsooften increased (Tables S1 and S2). It has been reported that proinflammatory cytokines such as TNF-a, IL-6 could modulate lipid metabolism by altering liver function and diminishing cholesterol efflux and transport[34]. In the COVID-19 infection, the SARS-CoV-2 pathogen may impair hepatic or renal function, leading to inhibition of lipid biosynthesis and transport; wherein inflammatory biomarkers were consistently associated with lipid metabolism, although but in the Wuhan cohort there was no significant association between many biomarkers of impaired organ function and lipid levels. Other complex biological and pathological processes induced by COVID-19 infection that affect lipid metabolism may remain to be discovered. Overall, the baseline lipid levels or prior to infections as possible may reflect the real metabolic condition, due to lipid metabolismderangement during thedisease course was associated with COVID-19 infections.

Patients discharged after testing negative for COVID-19 for a second time may still be at risk of recurrence, and thus could still be capable of spreading the virus[4]. Some patients test turned positive again when assessed by an oropharyngeal swab test for SARS-CoV-2 RNA. This could be due to a previous false-negative test result, which may occur due to sampling error, physician inexperience, or a low quantity of virus[35]. Performing multiple tests on from the same patient could be an effective approach to achieve more reliable SARS-CoV-2 RNA results[36]. To reduce the risk of COVID-19 transmission, recovering patients should be tested regularly, particularly if they have risk factors for relapse such as baseline dyslipidemia.

This is the first multi-center cohort study to investigate dyslipidemia as a possible risk factor for onset and recurrence of COVID-19; however, it had some limitations. First, although we adjusted for several common confounders, factors such as exercise and dietary habits were not taken into consideration. Second, data on recurrence of COVID-19 after discharge in the Wuhan cohort were not available, due to the heavy burden on the healthcare system during the early period of the outbreak. In contrast, Chengdu was not considered an endemic area so more medical resources were available to treat and follow up COVID-19 patients after discharge. Thirdly, the time between symptom onset and serum sample collection (i.e., hospital admission) varied among the patients. Therefore, lipid levels on admission may not accurately represent the baseline condition of patients prior to COVID-19 infection. Finally, a randomized clinical trial is required to identify the effect of lipid-lowering therapy on COVID-19 infection.Additional large-scale, well designed randomised controlled trials featuring good adherence to therapy are required.

\section{Conclusions}

This study provided robust evidence that dyslipidemia is a significant risk factor for ICU admissions, and with mortality and recurrence of COVID-19 after discharge. Further investigations were needed for COVID-19 patients with dyslipidemia should monitor them with statin therapy. Importantly, our findings also allude to a pathophysiological lipid profile in COVID-19, which enhance our understanding of the mechanisms underlying dyslipidemia.

\section{Declaration}

Ethics approval and consent to participate:The study was conducted in accordance with the Declaration of Helsinki and received approval from the local internal review board of the institutional ethics committee.

Consent for publication:Not applicable 
Availability of data and material:The datasets used and/or analysed during the current study are available from the corresponding author on reasonable request.

Competing interests $\square$ The authors declare no competing financial interests.

Funding: This research received the Youth Fund of Sichuan Medical Association (Q19018)

Authors' contributions: Xiaolong Zhao, Bin Li and Jiangang Fan had full access to all data in the study and took responsibility for the integrity of the data and the accuracy of the data. Study design: Yong Feng, Xiaoxu Yu, Xiaolong Zhao; Data collection: Hui Jing, Junji He, Tiande Huang, Luhong Cao, Bin Li; Statistical analysis: Xiaolong Zhao; Manuscript draft: Xiaolong Zhao, Zhiyue Ma.

Acknowledgments: The authors would like to thank all the subjects who participated in the study, and are also grateful to the Professor Peng He for the help in statistical analysis (Xuzhou Medical University School of Public Health).

\section{References}

1. Huang C, Wang Y, Li X, et al. Clinical features of patients infected with 2019 novel coronavirus in Wuhan, China. Lancet 2020; 395:497506.

2. World Health Organization. Novel Coronavirus (2019-nCoV) situation reports. https://www.who.int/emergencies/diseases/novelcoronavirus-2019/situation-reports/ (Assessed on April 14, 2020)

3. Chen D, Xu W, Lei Z, et al. Recurrence of positive SARS-CoV-2 RNA in COVID-19: A case report. Int J Infect Dis 2020; 93:297-299.

4. Hoang VT, Dao TL, Gautret P. Recurrence of positive SARS-CoV-2 in patients recovered from COVID-19. J Med Virol 2020.

5. Lakka HM, Laaksonen DE, Lakka TA, et al. The metabolic syndrome and total and cardiovascular disease mortality in middle-aged men. Jama 2002; 288:2709-2716.

6. Zhao X, Xu H, Qian Y, et al. Abdominal Obesity Is More Strongly Correlated with Obstructive Sleep Apnea than General Obesity in China: Results from Two Separated Observational and Longitudinal Studies. Obes Surg 2019; 29:2535-2547.

7. Zhao X, Li X, Xu H, et al. Relationships between cardiometabolic disorders and obstructive sleep apnea: Implications for cardiovascular disease risk. Journal of clinical hypertension 2019; 21:280-290.

8. Guan WJ, Liang WH, Zhao Y, et al. Comorbidity and its impact on 1590 patients with COVID-19 in China: a nationwide analysis. The European respiratory journal 2020; 55.

9. Mehra MR, Desai SS, Kuy S, et al. Cardiovascular Disease, Drug Therapy, and Mortality in Covid-19. The New England journal of medicine 2020.

10. Guo W, Li M, Dong Y, et al. Diabetes is a risk factor for the progression and prognosis of COVID-19. Diabetes/metabolism research and reviews 2020:e3319.

11. Chen N, Zhou M, Dong X, et al. Epidemiological and clinical characteristics of 99 cases of 2019 novel coronavirus pneumonia in Wuhan, China: a descriptive study. Lancet 2020; 395:507-513.

12. Cai Q, Chen F, Wang T, et al. Obesity and COVID-19 Severity in a Designated Hospital in Shenzhen, China. Diabetes care 2020.

13. Gao F, Zheng KI, Wang XB, et al. Obesity Is a Risk Factor for Greater COVID-19 Severity. Diabetes care 2020.

14. Wei X, Zeng W, Su J, et al. Hypolipidemia is associated with the severity of COVID-19. J Clin Lipidol 2020.

15. Fan J, Wang $\mathrm{H}, \mathrm{Ye} \mathrm{G}$, et al. Letter to the Editor: Low-density lipoprotein is a potential predictor of poor prognosis in patients with coronavirus disease 2019. Metabolism: clinical and experimental 2020:154243.

16. Radenkovic D, Chawla S, Pirro M, Sahebkar A, Banach M. Cholesterol in Relation to COVID-19: Should We Care about It? J Clin Med 2020, 9.

17. Diagnosis and Treatment Protocol for Novel Coronavirus Pneumonia (Trial Version 7). Chin Med J (Engl) 2020; 133:1087-1095. 
18. Zeng Y, Zhang B, Zhang X, Yi C. Clinical characteristics of 9 cancer patients with SARS-CoV-2 infection. Chin Med 2020; $15: 47$.

19. Gao HN, Lu HZ, Cao B, et al. Clinical findings in 111 cases of influenza A (H7N9) virus infection. The New England journal of medicine $2013 ; 368: 2277-2285$.

20. Stone NJ, Robinson JG, Lichtenstein AH, et al. 2013 ACC/AHA guideline on the treatment of blood cholesterol to reduce atherosclerotic cardiovascular risk in adults: a report of the American College of Cardiology/American Heart Association Task Force on Practice Guidelines. J Am Coll Cardiol 2014, 63:2889-2934.

21. Zhao X, Xu H, Zou J, et al. Joint interaction effect of metabolic syndrome and obstructive sleep apnea on hypertension. Journal of clinical hypertension 2018.

22. National Cholesterol Education Program Expert Panel on Detection E, Treatment of High Blood Cholesterol in A: Third Report of the National Cholesterol Education Program (NCEP) Expert Panel on Detection, Evaluation, and Treatment of High Blood Cholesterol in Adults (Adult Treatment Panel III) final report. Circulation 2002; 106:3143-3421.

23. Durrington PN. Triglycerides are more important in atherosclerosis than epidemiology has suggested. Atherosclerosis 1998,141 Suppl 1 : S57-62.

24. Lu Y, Liu DX, Tam JP. Lipid rafts are involved in SARS-CoV entry into Vero E6 cells. Biochem Biophys Res Commun $2008,369: 344-349$.

25. Jeon JH, Lee C. Cholesterol is important for the entry process of porcine deltacoronavirus. Arch Virol 2018, 163: $3119-3124$.

26. Madill J, Arendt BM, Aghdassi E, et al. Hepatic lipid peroxidation and antioxidant micronutrients in hepatitis virus $\mathrm{C}$ liver recipients with and without disease recurrence. Transplant Proc 2009; 41:3800-3805.

27. Morita M, Kuba K, Ichikawa A, et al. The lipid mediator protectin D1 inhibits influenza virus replication and improves severe influenza. Cell 2013; 153:112-125.

28. Katsiki N, Banach M, Mikhailidis DP. Lipid-lowering therapy and renin-angiotensin-aldosterone system inhibitors in the era of the COVID19 pandemic. Arch Med Sci 2020, 16:485-489.

29. Khademi F, Momtazi-Borojeni AA, Reiner Z, et al. PCSK9 and infection: A potentially useful or dangerous association? J Cell Physiol 2018, 233:2920-2927.

30. Bos LD, Schouten LR, van Vught LA, et al. Identification and validation of distinct biological phenotypes in patients with acute respiratory distress syndrome by cluster analysis. Thorax 2017, 72:876-883.

31. Tay MZ, Poh CM, Renia L, et al. The trinity of COVID-19: immunity, inflammation and intervention. Nat Rev Immunol 2020; $20: 363-374$.

32. Lima WG, Souza NA, Fernandes SOA, et al. Serum lipid profile as a predictor of dengue severity: A systematic review and meta-analysis. Rev Med Virol 2019; 29: e2056.

33. Cao WJ, Wang TT, Gao YF, et al. Serum Lipid Metabolic Derangement is Associated with Disease Progression During Chronic HBV Infection. Clin Lab 2019; 65.

34. Funderburg NT, Mehta NN. Lipid Abnormalities and Inflammation in HIV Inflection. Curr HIV/AIDS Rep 2016; 13:218-225.

35. Xiao AT, Tong YX, Zhang S. False-negative of RT-PCR and prolonged nucleic acid conversion in COVID-19: Rather than recurrence. J Med Virol 2020.

36. Pujadas E, Ibeh N, Hernandez MM, et al. Comparison of SARS-CoV-2 detection from nasopharyngeal swab samples by the Roche cobas 6800 SARS-CoV-2 test and a laboratory-developed real-time RT-PCR test. J Med Virol 2020.

\section{Tables}

Table 1. Demographic and clinical characteristics in the two cohorts. 


\begin{tabular}{|c|c|c|c|c|}
\hline & All patients & Chengdu cohort & Wuhan cohort & P-value \\
\hline & $\mathrm{n}=326$ & $\mathrm{n}=133$ & $\mathrm{n}=193$ & \\
\hline Age, years & $52.58 \pm 0.94$ & $44.99 \pm 1.48$ & $58.08 \pm 1.05$ & $P<0.001$ \\
\hline $\mathrm{BMI}, \mathrm{kg} / \mathrm{m} 2$ & $23.74 \pm 0.21$ & $23.36 \pm 0.34$ & $24.00 \pm 0.26$ & 0.132 \\
\hline No. of males, $\%$ & 52.6 & 51.4 & 53.3 & 0.731 \\
\hline Smoking status, $\%$ & 16.7 & 15.5 & 15.6 & 0.633 \\
\hline Drinking status, $\%$ & 19.9 & 23.9 & 17.8 & 0.231 \\
\hline \multicolumn{5}{|l|}{ Symptoms, \% } \\
\hline Fever on admission & $37.21 \pm 0.04$ & $36.9 \pm 0.05$ & $37.43 \pm 0.06$ & \multirow[t]{2}{*}{$P<0.001$} \\
\hline \multicolumn{4}{|l|}{ Temperature on admission } & \\
\hline$>37.3$ & 45 & 23.2 & 60.4 & $P<0.001$ \\
\hline Highest temperature $\left({ }^{\circ} \mathrm{C}\right)$ & $38.01 \pm 0.05$ & $37.67 \pm 0.06$ & $38.26 \pm 0.06$ & $P<0.001$ \\
\hline Nasal congestion & 0.5 & 0.8 & 0.6 & 0.866 \\
\hline Headache & 6.7 & 7.7 & 6.1 & 0.602 \\
\hline Cough & 77.5 & 60.6 & 89.3 & $P<0.001$ \\
\hline Sore throat & 11.1 & 13.4 & 9.6 & 0.347 \\
\hline Sputum production & 43 & 34.5 & 48.2 & 0.009 \\
\hline Fatigue & 41.5 & 23.2 & 53.8 & $P<0.001$ \\
\hline Shortness of breath & 38.3 & 12 & 56.3 & $P<0.001$ \\
\hline Nausea or vomiting & 1.8 & 0.7 & 2.5 & 0.194 \\
\hline Diarrhea & 12 & 3.5 & 18.3 & $P<0.001$ \\
\hline Chill & 8.5 & 8.5 & 8.6 & 0.886 \\
\hline \multicolumn{5}{|l|}{ Comorbidity, \% } \\
\hline Renal disease & 1.8 & 1.5 & 2 & 0.400 \\
\hline Pulmonary disease & 1.2 & 1.1 & 1.5 & 0.555 \\
\hline Heart disease & 5.7 & 5.4 & 5.9 & 0.908 \\
\hline Hepatic disease & 9.7 & 9.9 & 10.0 & 0.365 \\
\hline Intracranial disease & 2.3 & 2.8 & 2 & 0.638 \\
\hline Hypertension & 28.1 & 26.3 & 27.5 & 0.847 \\
\hline Obesity & 8.2 & 7.5 & 8.6 & 0.432 \\
\hline Diabetes & 9.1 & 8.8 & 9.6 & 0.698 \\
\hline Other diseases & 0.6 & 0.5 & 0.7 & 0.816 \\
\hline \multicolumn{5}{|l|}{ Lipid profiles on admission } \\
\hline $\mathrm{TC}, \mathrm{mmol} / \mathrm{L}$ & $3.97 \pm 0.06$ & $4.32 \pm 0.1$ & $3.72 \pm 0.06$ & $P<0.001$ \\
\hline TG, mmol/L & $1.46 \pm 0.05$ & $1.66 \pm 0.09$ & $1.31 \pm 0.04$ & $P<0.001$ \\
\hline $\mathrm{HDL}-\mathrm{C}, \mathrm{mmol} / \mathrm{L}$ & $1.07 \pm 0.02$ & $1.3 \pm 0.03$ & $0.9 \pm 0.02$ & $P<0.001$ \\
\hline LDL-C, mmol/L & $2.91 \pm 0.74$ & $2.86 \pm 0.76$ & $2.94 \pm 0.72$ & 0.322 \\
\hline
\end{tabular}


Disease severity, \%

Mild cases

Moderate cases

Severe cases

Critical cases

\section{The laboratory findings}

White blood cell count, 10ه/L

$<4$

4-10

$>10$

Neutrophil count, 10区/L

$<2$

2-7

$>7$

C-reactive protein, $>5 \mathrm{mg} / \mathrm{L}$

Lymphocyte count, 10ه/L

$<0.8$

$0.8-4$

$>4$

Platelet count, 10区/L

$<100$

$100-300$

$>300$

D-dimer, $>0.5 \mathrm{mg} / \mathrm{L}$

Alanine aminotransferase, $>40 \mathrm{U} / \mathrm{L}$

Aspartate aminotransferase, $>40 \mathrm{U} / \mathrm{L}$

Creatinine, $\mu \mathrm{mol} / \mathrm{L}$

$<40$

40-133

$>133$

Lactate dehydrogenase, U/L

$<109$

$109-245$

$>109$

High-sensitive cardiac

troponin I, ng/mL

Procalcitonin, $>0.5 \mu \mathrm{g} / \mathrm{L}$
6.4

59.1

11.1

23.4

11.1

63

11.9

14.1

59.4

11.2

29.4 


\begin{tabular}{|c|c|c|c|c|}
\hline Fasting glucose, $\mathrm{mmol} / \mathrm{L}$ & $6.19 \pm 0.13$ & $5.55 \pm 0.09$ & $6.66 \pm 0.21$ & $P<0.001$ \\
\hline Imaging features, \% & & & & $P<0.001$ \\
\hline Normal & 0.6 & 1.4 & 0 & \\
\hline Local patchy shadowing & 13.5 & 24.6 & 5.6 & \\
\hline Bilateral patchy shadowing & 85.9 & 71.8 & 94.4 & \\
\hline \multicolumn{5}{|l|}{ Treatments, \% } \\
\hline Antibiotics & 64.9 & 23.2 & 94.4 & $P<0.001$ \\
\hline Antiviral treatment & 98.0 & 99.3 & 97 & 0.135 \\
\hline Corticosteroids & 17.7 & 8.9 & 65.2 & $P<0.001$ \\
\hline Intravenous immunoglobin & 25.1 & 1.4 & 41.4 & $P<0.001$ \\
\hline Antifungal medications & 1.8 & 2.1 & 1 & 0.408 \\
\hline Oxygen therapy & 87.7 & 98.6 & 79.7 & $P<0.001$ \\
\hline Mechanical ventilation & 21.3 & 11.3 & 27.4 & $P<0.001$ \\
\hline Extracorporeal membrane oxygenation & 1.4 & 1.4 & 0 & NS \\
\hline ICU admission & 28.9 & 42.3 & 18.3 & $P<0.001$ \\
\hline \multicolumn{5}{|l|}{ Lipid-lowering therapy } \\
\hline Statin therapy, \% & 22.08 & 18.8 & 24.4 & 0.235 \\
\hline High-intensity statin therapy, \% & & $7 / 5.26$ & $13 / 6.73$ & 0.421 \\
\hline Moderate-intensity statin therapy, \% & & $10 / 7.51$ & $20 / 10.36$ & \\
\hline Low-intensity statin therapy, \% & & $8 / 6.01$ & $14 / 7.25$ & \\
\hline \multicolumn{5}{|l|}{ Disease period } \\
\hline $\begin{array}{l}\text { Time from symptom onset to } \\
\text { first hospital admission, days }\end{array}$ & $7.84 \pm 5.31$ & $6.22 \pm 6.51$ & $9 \pm 5.36$ & $P<0.001$ \\
\hline The first hospital length of stay, days & $17.46 \pm 10.57$ & $20.53 \pm 12.05$ & $15.25 \pm 7.57$ & $P<0.001$ \\
\hline The second hospital length of stay, days & & $14.2 \pm 6.68$ & NS & NS \\
\hline $\begin{array}{l}\text { The recurrence time from } \\
\text { the first discharge }\end{array}$ & & $18.22 \pm 7.07$ & NS & NS \\
\hline Time from symptom onset to the endpoint, days & $29.63 \pm 16.91$ & $37.11 \pm 21.8$ & $24.25 \pm 8.64$ & $P<0.001$ \\
\hline \multicolumn{5}{|l|}{ Complications, \% } \\
\hline Septic shock & 3.6 & 0.7 & 5.6 & 0.020 \\
\hline Acute respiratory distress syndrome (ARDS) & 10.08 & 11.1 & 10.8 & 0.915 \\
\hline Acute kidney injury & 1.2 & 0.7 & 1.5 & 0.491 \\
\hline Disseminated intravascular coagulation & 0.3 & 0.7 & 0 & 0.238 \\
\hline Pneumonia & 80.5 & 69.7 & 92.9 & $P<0.001$ \\
\hline Multiple organ failure & 0.9 & 0 & 1.5 & 0.341 \\
\hline Clinical endpoints, \% & & & & 0.001 \\
\hline Deaths in hospital & & & 28.9 & \\
\hline
\end{tabular}

Page $11 / 15$ 
Continuous data are presented as the means \pm standard deviation (SD) and categorical data are presented as percentages.

Differences in baseline characteristics among the subgroups were examined using the Kruskal-Wallis $\mathrm{H}$ test or $\chi 2$ test according to the characteristics of the data distribution.

Abbreviations: BMI, body mass index; ICU, intensive care unit; TC, total cholesterol; TG, triglycerides; HDL-C, high-density lipoprotein cholesterol; LDL-C, low-density lipoprotein cholesterol.

Table 2. Risk factors associated with re-positive SARS-CoV-2 RNA detection after first discharge or in-hospital death.

\begin{tabular}{|c|c|c|c|c|}
\hline & Univariable RR $(95 \% \mathrm{Cl})$ & $\mathrm{p}$ value & Multivariable RR $(95 \% \mathrm{Cl})$ & $p$ value \\
\hline \multicolumn{5}{|l|}{ Chengdu cohort } \\
\hline TC dyslipidemia (vs not present) & $1.335(1.014,2.495)$ & 0.008 & & \\
\hline TG dyslipidemia (vs not present) & $0.856(0.468,1.568)$ & 0.615 & & \\
\hline HDL-c dyslipidemia (vs not present) & $0.037(0.000,4.883)$ & 0.186 & & \\
\hline LDL-c dyslipidemia (vs not present) & $2.708(1.283,5.718)$ & 0.009 & $2.51(1.19,5.29)$ & 0.006 \\
\hline Statin therapy (vs not present) & $1.609(0.771,3.355)$ & 0.205 & & \\
\hline \multicolumn{5}{|l|}{ Wuhan cohort } \\
\hline TC dyslipidemia (vs not present) & $1.655(0.746,3.674)$ & 0.216 & & \\
\hline TG dyslipidemia (vs not present) & $2.428(1.407,4.189)$ & 0.001 & $1.92(1.08,3.60)$ & 0.016 \\
\hline HDL-c dyslipidemia (vs not present) & $1.535(0.841,2.802)$ & 0.163 & & \\
\hline LDL-c dyslipidemia (vs not present) & $1.403(0.629,3.131)$ & 0.408 & & \\
\hline Statin therapy (vs not present) & $0.849(0.460,1.564)$ & 0.599 & & \\
\hline
\end{tabular}

The data are presented as the risk ratio $(\mathrm{RR})(95 \% \mathrm{Cl})$. We performed Cox proportional hazards regression to determine the potential risk factors associated with the endpoint during time from illness onset to follow-up.

The RRs for endpoints were adjusted for age (continuous variables), BMI, males (vs female), smoking status (vs not present), and drinking status (vs not present) and various kind of comorbidities (eg, chronic renal disease, chronic pulmonary disease, hypertension, heart disease, hepatic disease), and laboratory findings (eg, lymphopenia, and elevated white blood cell count, ALT, AST, high lactate dehydrogenase, highsensitivity cardiac troponin I, d-dimer, C-reactive protein, creatinine, and procalcitonin) and treatments in hospital .

Abbreviations: ALT, alanine aminotransferase; AST, aspartate aminotransferase; TC, total cholesterol; TG, triglycerides; HDL-C, high-density lipoprotein cholesterol; LDL-C, low-density lipoprotein cholesterol.

Table 3. Risk factors associated with the composite clinical outcomes in hospital. 


\begin{tabular}{|c|c|c|c|c|c|c|c|c|}
\hline & \multicolumn{4}{|c|}{ Mechanical ventilation } & \multicolumn{4}{|c|}{ Intensive care unit admission } \\
\hline & $\begin{array}{l}\text { Univariable OR } \\
(95 \% \mathrm{Cl})\end{array}$ & $\begin{array}{l}\mathrm{p} \\
\text { value }\end{array}$ & $\begin{array}{l}\text { Multivariable } \\
\text { OR }(95 \% \mathrm{Cl})\end{array}$ & $\begin{array}{l}\mathrm{p} \\
\text { value }\end{array}$ & $\begin{array}{l}\text { Univariable OR } \\
(95 \% \mathrm{Cl})\end{array}$ & $\begin{array}{l}\mathrm{p} \\
\text { value }\end{array}$ & $\begin{array}{l}\text { Multivariable } \\
\text { OR }(95 \% \mathrm{Cl})\end{array}$ & $\begin{array}{l}\mathrm{p} \\
\text { value }\end{array}$ \\
\hline \multicolumn{9}{|l|}{$\begin{array}{l}\text { Chengdu } \\
\text { cohort }\end{array}$} \\
\hline $\begin{array}{l}\text { TC } \\
\text { dyslipidemia } \\
\text { (vs not } \\
\text { present) }\end{array}$ & $0.436(0.053,3.6)$ & 0.441 & & & $3.574(1.452,8.797)$ & 0.006 & & \\
\hline $\begin{array}{l}\text { TG } \\
\text { dyslipidemia } \\
\text { (vs not } \\
\text { present) }\end{array}$ & $0.518(0.105,2.551)$ & 0.419 & & & $1.312(0.630,2.732)$ & 0.468 & & \\
\hline $\begin{array}{l}\text { HDL-C } \\
\text { dyslipidemia } \\
\text { (vs not } \\
\text { present) }\end{array}$ & $0.865(0.102,7.350)$ & 0.894 & & & $0.195(0.042,0.904)$ & 0.057 & & \\
\hline $\begin{array}{l}\text { LDL-C } \\
\text { dyslipidemia } \\
\text { (vs not } \\
\text { present) }\end{array}$ & & & & & $9.849(2.086,46.503)$ & 0.004 & $10.073(2.073,48.948)$ & 0.004 \\
\hline $\begin{array}{l}\text { Statin } \\
\text { therapy (vs } \\
\text { not present) }\end{array}$ & $0.865(0.102,7.35)$ & 0.894 & & & $3.364(1.080,10.481)$ & 0.036 & & \\
\hline \multicolumn{9}{|l|}{$\begin{array}{l}\text { Wuhan } \\
\text { cohort }\end{array}$} \\
\hline $\begin{array}{l}\text { TC } \\
\text { dyslipidemia } \\
\text { (vs not } \\
\text { present) }\end{array}$ & $1.970(0.665,5.842)$ & 0.221 & & & $2.569(1.218,8.072)$ & 0.016 & $8.147(1.662,39.927)$ & 0.010 \\
\hline $\begin{array}{l}\text { TG } \\
\text { dyslipidemia } \\
\text { (vs not } \\
\text { present) }\end{array}$ & $1.427(0.671,3.035)$ & 0.356 & & & $2.090(0.922,4.735)$ & 0.077 & & \\
\hline $\begin{array}{l}\text { HDL-C } \\
\text { dyslipidemia } \\
\text { (vs not } \\
\text { present) }\end{array}$ & $1.266(0.651,2.461)$ & 0.487 & & & $1.060(0.495,2.269)$ & 0.881 & & \\
\hline $\begin{array}{l}\text { LDL-C } \\
\text { dyslipidemia } \\
\text { (vs not } \\
\text { present) }\end{array}$ & $1.970(0.665,5.842)$ & 0.221 & & & $2.569(0.818,8.072)$ & 0.106 & & \\
\hline $\begin{array}{l}\text { Statin } \\
\text { therapy (vs } \\
\text { not present) }\end{array}$ & $0.959(0.476,1.933)$ & 0.907 & & & $1.140(0.515,2.524)$ & 0.747 & & \\
\hline
\end{tabular}

The data are presented as the odds ratio $(O R)(95 \% \mathrm{Cl})$. We performed logistic regression model to determine the potential risk factors associated with the composite clinical outcomes in hospital.

The ORs for endpoints were adjusted for age (continuous variables), BMI, males (vs female), smoking status (vs not present), and drinking status (vs not present) and various kind of comorbidities (eg, chronic renal disease, pulmonary disease, hypertension, heart disease, hepatic disease), and laboratory findings (eg, lymphopenia, and elevated white blood cell count, ALT, AST, high lactate dehydrogenase, highsensitivity cardiac troponin I, d-dimer, C-reactive protein, creatinine, and procalcitonin) and treatments in hospital.

Abbreviations: ALT, alanine aminotransferase; AST, aspartate aminotransferase; TC, total cholesterol; TG, triglycerides; HDL-C, high-density lipoprotein cholesterol; LDL-C, low-density lipoprotein cholesterol. 
Table 4. The multivariate linear regression model for lipid level on admission in COVID -19 patients for two cohorts respectively.

\begin{tabular}{|c|c|c|c|c|c|c|c|c|}
\hline & $\mathrm{TC}$ & $\begin{array}{l}\mathrm{p} \\
\text { value }\end{array}$ & TG & $\begin{array}{l}\mathrm{p} \\
\text { value }\end{array}$ & HDL & $\begin{array}{l}\mathrm{p} \\
\text { value }\end{array}$ & LDL & $\begin{array}{l}\mathrm{p} \\
\text { value }\end{array}$ \\
\hline \multicolumn{9}{|l|}{ Chengdu cohort } \\
\hline C-reactive protein, $\mathrm{mg} / \mathrm{L}$ & $-0.646(0.219)$ & 0.005 & & & $-0.224(0.049)$ & 0.001 & & \\
\hline \multicolumn{9}{|l|}{ Procalcitonin, $\mu \mathrm{g} / \mathrm{L}$} \\
\hline Alanine aminotransferase, $\mathrm{U} / \mathrm{L}$ & $0.633(0.229)$ & 0.007 & & & & & $0.524(0.144)$ & 0.001 \\
\hline $\begin{array}{l}\text { Aspartate aminotransferase, } \\
\text { U/L }\end{array}$ & & & & & $0.173(0.075)$ & 0.023 & & \\
\hline \multicolumn{9}{|l|}{ Creatinine, $\mu \mathrm{mol} / \mathrm{L}$} \\
\hline \multicolumn{9}{|l|}{ Lactate dehydrogenase, U/L } \\
\hline \multicolumn{9}{|l|}{$\begin{array}{l}\text { High-sensitive cardiac } \\
\text { troponin I, ng/mL }\end{array}$} \\
\hline \multicolumn{9}{|l|}{ Wuhan cohort } \\
\hline \multicolumn{9}{|l|}{ C-reactive protein, mg/L } \\
\hline Procalcitonin, $\mu \mathrm{g} / \mathrm{L}$ & $-0.338(0.135)$ & 0.013 & & & $-0.163(0.040)$ & 0.001 & $-0.251(0.096)$ & 0.010 \\
\hline Alanine aminotransferase, $\mathrm{U} / \mathrm{L}$ & & & $0.169(0.085)$ & 0.048 & & & & \\
\hline \multicolumn{9}{|l|}{$\begin{array}{l}\text { Aspartate aminotransferase, } \\
\mathrm{U} / \mathrm{L}\end{array}$} \\
\hline Creatinine, $\mu \mathrm{mol} / \mathrm{L}$ & & & $0.244(0.056)$ & 0.001 & & & & \\
\hline \multicolumn{9}{|l|}{ Lactate dehydrogenase, U/L } \\
\hline $\begin{array}{l}\text { High-sensitive cardiac } \\
\text { troponin I, ng/mL }\end{array}$ & & & & & & & & \\
\hline
\end{tabular}

The data are presented as $\beta$ (SE). The multivariate linear regression model was adjusted for age, BMI, males (vs female), smoking and drinking status (vs not present) and various kind of comorbidities (eg, hypertension, chronic renal disease, pulmonary disease, heart disease, hepatic disease) and the use of lipid-lowering therapy.

Abbreviations: TC, total cholesterol; TG, triglycerides; HDL-C, high-density lipoprotein cholesterol; LDL-C, low-density lipoprotein cholesterol.

\section{Figures}




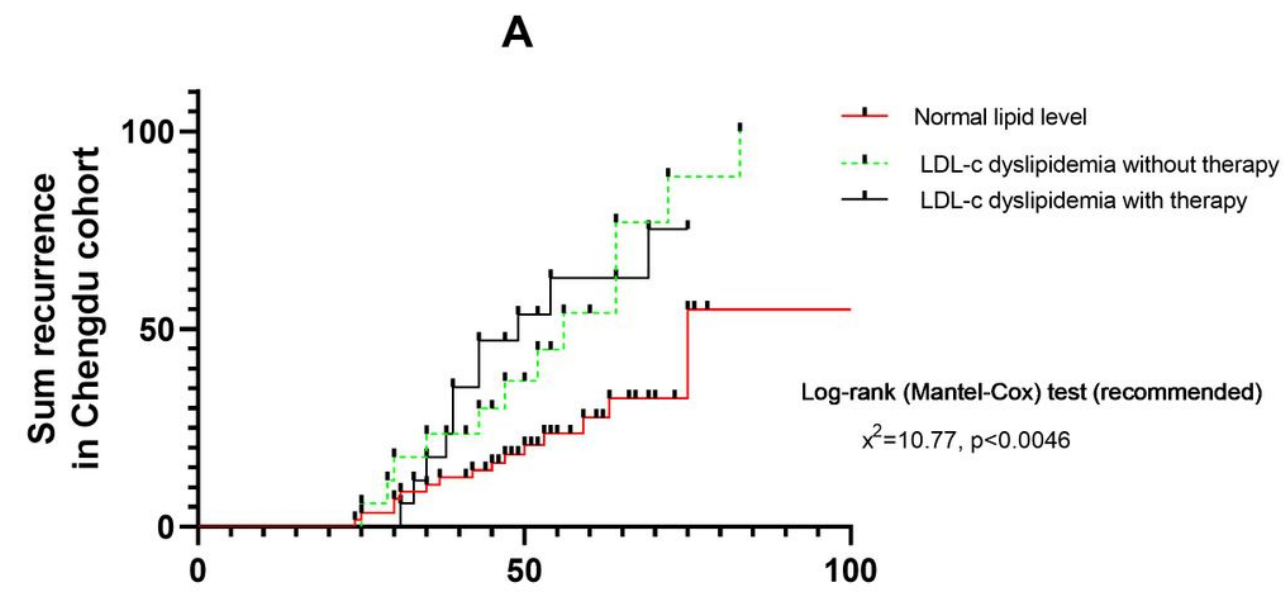

The time from symptom onset to recurrence (Days)

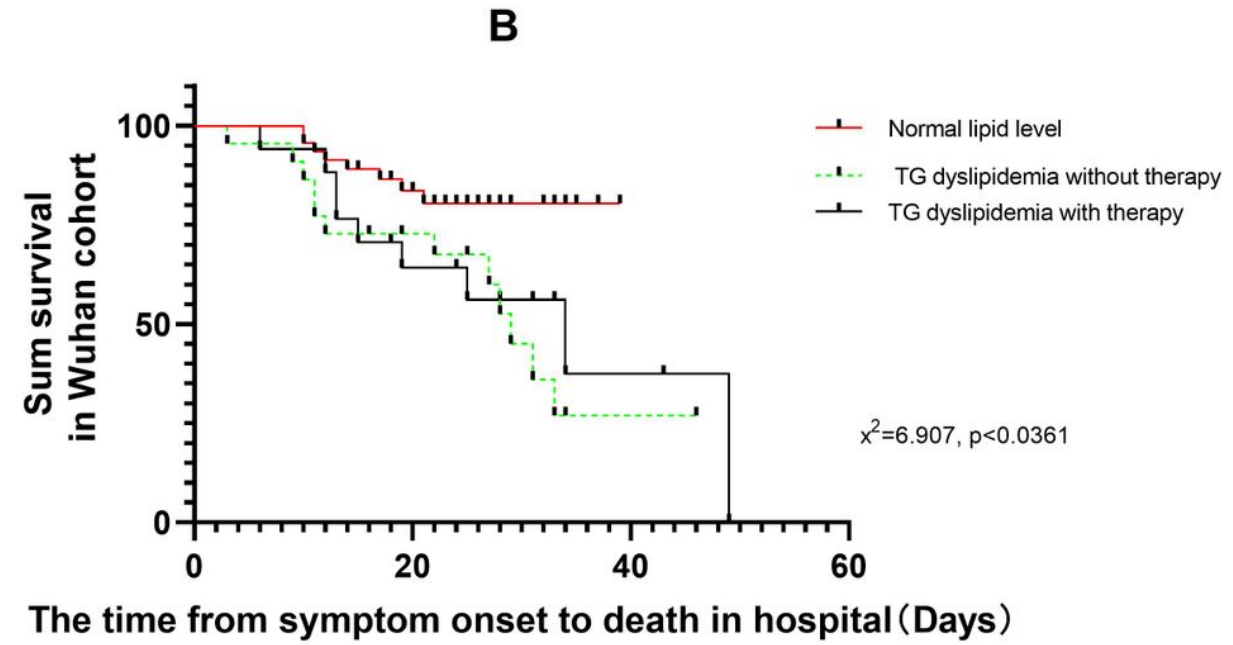

Figure 1

Comparison of the time-dependent risk of reaching to the recurrence after discharge or in-hospital death. The time-dependent risk of reaching to the recurrence after discharge in COVID-19 patients with dyslipidemia or statins therapy compared with the control in the Chengdu cohort (Figure 1A). Figure 1B showed that the time-dependent risk of reaching to the in-hospital death in COVID-19 patients with dyslipidemia or statins therapy compared with the control in the Wuhan cohort. Kaplan-Meier analysis was used to estimate and plot survival outcomes among subgroups, with the log-rank test being reported. The English in this document has been checked by at least two professional editors, both native speakers of English. For a certificate, please see: http://www.textcheck.com/certificate/dxHFjW

\section{Supplementary Files}

This is a list of supplementary files associated with this preprint. Click to download.

- Tables2.doc

- Tables2.doc

- Tables2.doc

- Tables1.doc

- Tables1.doc

- Tables1.doc 